\title{
On intervals containing full sets of conjugates of algebraic integers
}

\author{
by
}

\author{
Artūras Dubickas (Vilnius)
}

1. Introduction. Let $\alpha$ be an algebraic number with $a\left(x-\alpha_{1}\right) \ldots$ $\ldots\left(x-\alpha_{d}\right)$ as its minimal polynomial over $\mathbb{Z}$. Then $\alpha$ is called totally real if all its conjugates $\alpha_{1}=\alpha, \alpha_{2}, \ldots, \alpha_{d}$ are real. Also, $\alpha$ is called an algebraic integer if $a=1$. Now, define $I(d)$ as the smallest positive number with the following property: any closed real interval of length at least $I(d)$ contains a full set of conjugates of an algebraic integer of degree $d$. It is clear that $I(1)=1$.

THEOREM 1. We have

$$
I(2)=\frac{1+\sqrt{5}}{2}+\sqrt{2} .
$$

As we will see from the proof of this simple theorem $I(d)$ can also be computed for all small $d$. The purpose of this paper is to give an upper bound for $I(d)$ for large $d$.

In 1918, I. Schur [Sc] proved that an interval on the real axis of length smaller than 4 can contain only a finite number of full sets of conjugates of algebraic integers. T. Zaïmi $[\mathrm{Za}]$ gave another proof of Schur's result. His approach is based on M. Langevin's proof [La] of Favard's conjecture. Moreover, in $[\mathrm{Za}]$ it is proved that the length of an interval containing a full set of conjugates of an algebraic integer of degree $d$ is greater than $4-\psi_{1}(d)$ with some explicitly given positive function $\psi_{1}(d)$ satisfying $\lim _{d \rightarrow \infty} \psi_{1}(d)=0$. Note that a similar result with another explicitly given function $\psi_{2}(d)$ also follows from $[\mathrm{Sc}]$.

On the other hand, R. Robinson [Ro] showed that any interval of length greater than 4 contains infinitely many full sets of conjugates of algebraic integers. Moreover, V. Ennola [En] proved that such an interval contains full sets of conjugates of algebraic integers of degree $d$ for all $d$ sufficiently large. Hence $\lim _{d \rightarrow \infty} I(d)=4$.

1991 Mathematics Subject Classification: 11R04, 11R80, 12D10. 
A lower bound for $I(d)$ can be obtained via a Kronecker type theorem. In $1857, \mathrm{~L}$. Kronecker $[\mathrm{Kr}]$ proved that if $\alpha$ is an algebraic integer all of whose conjugates lie in $[-2 ; 2]$ then $\alpha=2 \cos (\pi r)$ with $r$ rational. So if $\alpha$ is a totally real algebraic integer not of the form $2 \cos (\pi r)$ with $r$ rational, then

$$
|\alpha|=\max _{1 \leq j \leq d}\left|\alpha_{j}\right|>2 .
$$

In 1965, A. Schinzel and H. Zassenhaus [SZ] asked for a lower bound of the house $\sqrt{\alpha}$ in terms of the degree $d$ of $\alpha$. They showed that with the same hypotheses,

$$
|\alpha|>2+4^{-2 d-3} \text {. }
$$

This lower bound was derived from the lower bound for $\alpha$, where $\alpha$ is an algebraic integer which is not a root of unity. The conjectural inequality $|\alpha|>1+c_{1} / d$ (see [SZ]) with an absolute positive constant $c_{1}$ is not yet proved. This is also the case with D. H. Lehmer's [Le] more general conjectural inequality

$$
M(\alpha)=a \prod_{j=1}^{d} \max \left\{1,\left|\alpha_{j}\right|\right\}>1+c_{2}
$$

where $\alpha$ is an algebraic number which is not a root of unity and $c_{2}$ is an absolute positive constant. Using the best known lower bound in Lehmer's conjecture [Lo] the author strengthened the inequality (1). We proved [Du] that if $\alpha$ is a totally real algebraic integer of degree $d, \alpha \neq 2 \cos (\pi r)$ with $r$ rational, and $d$ is sufficiently large, then

Thus the interval

$$
\mid \alpha>2+4.6 \frac{(\log \log d)^{3}}{d(\log d)^{4}} .
$$

$$
\left(-2 \cos \left(\frac{\pi}{2 d}\right) ; 2+4.6 \frac{(\log \log d)^{3}}{d(\log d)^{4}}\right]
$$

does not contain a full set of conjugates of an algebraic integer of degree $d$. It follows immediately that for all $d$ sufficiently large,

$$
I(d)>4+\frac{9}{2} \cdot \frac{(\log \log d)^{3}}{d(\log d)^{4}} .
$$

Our main theorem gives an explicit slowly decreasing function, namely $12(\log \log d)^{2} / \log d$, which cannot replace $9(\log \log d)^{3} / 2 d(\log d)^{4}$ in (2).

THEOREM 2. There is an infinite sequence $S$ of positive integers such that for $d \in S$ any interval of length greater than or equal to

$$
4+12 \frac{(\log \log d)^{2}}{\log d}
$$

contains a full set of conjugates of an algebraic integer of degree $d$. 
Clearly, for $d \in S$ we have the inequality

$$
I(d) \leq 4+12 \frac{(\log \log d)^{2}}{\log d} .
$$

Our proof of Theorem 2 is based on the following statement.

Lemma. Let $u, v, w$ be three fixed positive integers. Then there is an infinite sequence $S(u, v, w)$ of positive integers such that every $d \in S(u, v, w)$ is divisible by

$$
w\left(v q(d)^{u}\right)^{q(d)} q(d) !
$$

where

$$
q(d)=\left[\frac{\log d}{(u+1) \log \log d}\right] .
$$

Here and below $[. .$.$] denotes the integral part. We will also show that the$ sequence $S$ in Theorem 2 can be taken to be all sufficiently large elements of $S(2,16,2)$.

Now we will prove the Lemma, Theorem 2 and Theorem 1.

2. Proof of the Lemma. Put for brevity

$$
f(x)=\frac{\log x}{\log \log x} .
$$

For $x \geq 16$ the function $f(x)$ is increasing. Let $k \geq 2$ be an integer. Then the equation (in $x$ )

$$
\frac{f(x)}{u+1}=k
$$

has a unique solution which we denote by $x_{k}$. Clearly, $x_{2}>5503$ and the sequence $x_{k}$ is increasing. We now prove that

$$
x_{k+1}>x_{k} \log x_{k} .
$$

Indeed, if $x_{k+1} \leq x_{k} \log x_{k}$ then

$$
\begin{aligned}
u+1 & =(u+1)(k+1)-(u+1) k=f\left(x_{k+1}\right)-f\left(x_{k}\right) \\
& \leq f\left(x_{k} \log x_{k}\right)-f\left(x_{k}\right)=\frac{\log \left(x_{k} \log x_{k}\right)}{\log \log \left(x_{k} \log x_{k}\right)}-\frac{\log x_{k}}{\log \log x_{k}} .
\end{aligned}
$$

Put $y_{k}=\log \log x_{k}$ for brevity. By the last inequality we have

$$
\begin{aligned}
2 & \leq u+1 \leq \frac{y_{k}+e^{y_{k}}}{\log \left(y_{k}+e^{y_{k}}\right)}-\frac{e^{y_{k}}}{y_{k}}=\frac{y_{k}^{2}+y_{k} e^{y_{k}}-e^{y_{k}} \log \left(y_{k}+e^{y_{k}}\right)}{y_{k} \log \left(y_{k}+e^{y_{k}}\right)} \\
& =\frac{y_{k}^{2}-e^{y_{k}} \log \left(1+y_{k} e^{-y_{k}}\right)}{y_{k} \log \left(y_{k}+e^{y_{k}}\right)}<\frac{y_{k}^{2}-e^{y_{k}} \log \left(1+y_{k} e^{-y_{k}}\right)}{y_{k}^{2}} .
\end{aligned}
$$

The last expression is less than 1 , a contradiction. 
Set

$$
N_{k}=\left\{\left[x_{k}\right]+1,\left[x_{k}\right]+2, \ldots,\left[x_{k} \log x_{k}\right]\right\} .
$$

Clearly, for $n \in N_{k}$,

$$
q(n)=\left[\frac{f(n)}{u+1}\right]=k .
$$

Note that for all $n$ sufficiently large the expression

$$
r(n)=w\left(v q(n)^{u}\right)^{q(n)} q(n) !
$$

is less than

$$
\begin{aligned}
w\left(v q(n)^{u}\right)^{q(n)} q(n)^{q(n)} \leq & \exp \left(\log w+\frac{\log v \log n}{(u+1) \log \log n}\right. \\
& \left.+\frac{\log n}{\log \log n} \log \left(\frac{\log n}{(u+1) \log \log n}\right)\right) \\
< & \exp (\log n)=n,
\end{aligned}
$$

so that for all $n \in N_{k}$,

$$
r(n)=w\left(v k^{u}\right)^{k} k ! \leq\left[x_{k}\right] .
$$

Hence, at least one of the integers $\left[x_{k}\right]+1,\left[x_{k}\right]+2, \ldots, 2\left[x_{k}\right]$ is divisible by $w\left(v k^{u}\right)^{k} k$ !. Since $2\left[x_{k}\right] \leq\left[x_{k} \log x_{k}\right]$, at least one element of $N_{k}$ belongs to $S(u, v, w)$. The Lemma is proved.

3. Proof of Theorem 2. Let $d$ be a sufficiently large positive integer from $S(2,16,2)$. Suppose $[A ; B]$ is a real interval such that

$$
B-A \geq 4+12 \frac{(\log \log d)^{2}}{\log d} .
$$

Let also

$$
q=\left[\frac{\log d}{3 \log \log d}\right] .
$$

We take two integers $p_{1}$ and $p_{2}$ in the intervals

$$
\left[A q ;\left(A+\frac{4 \log \log d}{\log d}\right) q\right) \text { and }\left(\left(B-\frac{4 \log \log d}{\log d}\right) q ; B q\right]
$$

respectively. Then $\left[p_{1} / q ; p_{2} / q\right] \subset[A ; B]$ and

$$
\frac{p_{2}}{q}-\frac{p_{1}}{q} \geq B-A-\frac{8 \log \log d}{\log d}>4+12 \frac{(\log \log d)^{2}-\log \log d}{\log d} .
$$

We will show that the interval $\left[p_{1} / q ; p_{2} / q\right]$ contains a full set of conjugates of an algebraic integer of degree $d$. Define

$$
\varrho=\frac{p_{1}+p_{2}}{2 q}, \quad \lambda=\frac{p_{2}-p_{1}}{4 q} .
$$


Following [Ro] and [En] an irreducible monic polynomial of degree $d$ with all $d$ roots in the interval $\left[p_{1} / q ; p_{2} / q\right]=[\varrho-2 \lambda ; \varrho+2 \lambda]$ can be constructed by means of the Chebyshev polynomials

$$
T_{m}(x)=x^{m}+\sum_{j=1}^{[m / 2]}(-1)^{j} \frac{m}{j 4^{j}}\left(\begin{array}{c}
m-j-1 \\
j-1
\end{array}\right) x^{m-2 j} .
$$

In $[-1 ; 1]$ these are also given by the formula

$$
T_{m}(x)=2^{1-m} \cos (m \arccos x) .
$$

Set

We write

$$
P_{m}(x)=(2 \lambda)^{m} T_{m}\left(\frac{x-\varrho}{2 \lambda}\right)
$$

$$
P_{d}(x)=x^{d}+\sum_{j=1}^{d} c_{d, j} x^{d-j} .
$$

The denominators of the rational numbers $\varrho$ and $2 \lambda$ are both at most $2 q$. Hence the coefficients $c_{d, 1}, c_{d, 2}, \ldots, c_{d, q}$ are all even integers if $d$ is divisible by $2 q ! 4^{q}(2 q)^{2 q}$. This is exactly the case, since $d \in S(2,16,2)$ (see the Lemma). All the polynomials $P_{m}(x)$ are monic, except for $P_{0}(x)=2$. So in $[-1 ; 1)$ there are numbers $b_{q+1}, b_{q+2}, \ldots, b_{d}$ such that the polynomial

$$
Q_{d}(x)=P_{d}(x)+\sum_{j=1+q}^{d} b_{j} P_{d-j}(x)=x^{d}+\sum_{j=0}^{d-1} a_{j} x^{j}
$$

has all coefficients $a_{k}$ integral and even, $a_{0}$ not being divisible by 4 . Therefore, $Q_{d}(x)$ is irreducible by Eisenstein's criterion.

In the interval $[\varrho-2 \lambda ; \varrho+2 \lambda]$ the maximum of the absolute value of $P_{m}(x)$ equals $2 \lambda^{m}$. Consequently, in this interval we bound

$$
\left|Q_{d}(x)-P_{d}(x)\right| \leq 2 \sum_{j=1+q}^{d} \lambda^{d-j}=2 \frac{\lambda^{d-q}-1}{\lambda-1}<\frac{2 \lambda^{d}}{\lambda^{q}(\lambda-1)} .
$$

Since

$$
q>\frac{\log d}{3 \log \log d}-1 \quad \text { and } \quad \lambda>1+3 \frac{(\log \log d)^{2}-\log \log d}{\log d},
$$

for large $d$ we have

$$
\begin{aligned}
q \log \lambda & >q(\lambda-1)\left(1-\frac{\lambda-1}{2}\right) \\
& >\left(\log \log d-1-\frac{3(\log \log d)^{2}}{\log d}\right)\left(1-\frac{3(\log \log d)^{2}}{2 \log d}\right) \\
& >\log \log d-2 .
\end{aligned}
$$


Therefore,

$$
\lambda^{q}(\lambda-1)>\frac{\log d}{e^{2}}(\lambda-1)>\frac{(\log \log d)^{2}}{3}>1 .
$$

Hence, in the interval $\varrho-2 \lambda \leq x \leq \varrho+2 \lambda$ we have

$$
\left|Q_{d}(x)-P_{d}(x)\right|<2 \lambda^{d} .
$$

Suppose $\xi_{1}<\ldots<\xi_{d+1}$ are the points in $[\varrho-2 \lambda ; \varrho+2 \lambda]$ such that $\left|P_{d}\left(\xi_{j}\right)\right|=2 \lambda^{d}$. By our choice, $d$ is even. So $P_{d}\left(\xi_{j}\right)$ is positive for odd $j$ and negative for even $j$. From (3) we see that at each of the points $\xi_{1}, \ldots, \xi_{d+1}$ the signs of the values of $Q_{d}(x)$ and $P_{d}(x)$ coincide. Hence in each of the $d$ intervals $\left(\xi_{i} ; \xi_{i+1}\right)$, where $i=1, \ldots, d$, there is a zero of $Q_{d}(x)$. This proves Theorem 2 .

4. Proof of Theorem 1. We first prove that

$$
I(2) \geq \frac{1+\sqrt{5}}{2}+\sqrt{2} .
$$

It suffices to show that no interval $[A ; B]$ with $A>1-\sqrt{2}, B<(3+\sqrt{5}) / 2$ contains both conjugates of an algebraic integer of degree two.

Indeed, suppose that

$$
\begin{aligned}
& \frac{p-\sqrt{p^{2}-4 q}}{2}>1-\sqrt{2}, \\
& \frac{p+\sqrt{p^{2}-4 q}}{2}<\frac{3+\sqrt{5}}{2}
\end{aligned}
$$

with integers $p, q$ such that $p^{2}-4 q$ is a positive integer which is not a perfect square. Then

$$
\sqrt{p^{2}-4 q}<\frac{3+\sqrt{5}}{2}-1+\sqrt{2}=\frac{1+\sqrt{5}}{2}+\sqrt{2}<3.1,
$$

so that $p^{2}-4 q \leq 9$. Since $p^{2}-4 q$ modulo 4 is zero or one and $p^{2}-4 q$ is not a perfect square, it equals 5 or 8 . In the case $p^{2}-4 q=5$ inequality (5) implies that $p \leq 2$. Also, $p$ is odd, hence $p \leq 1$. Then

$$
\frac{p-\sqrt{p^{2}-4 q}}{2} \leq \frac{1-\sqrt{5}}{2}<1-\sqrt{2},
$$

which contradicts (4).

If $p^{2}-4 q=8$, then inequality (4) implies that $p>2$. Hence $p \geq 4$, since $p$ is even. Then

$$
\frac{p+\sqrt{p^{2}-4 q}}{2} \geq \frac{4+\sqrt{8}}{2}>\frac{3+\sqrt{5}}{2},
$$

which contradicts (5). 
To prove the inequality

$$
I(2) \leq \frac{1+\sqrt{5}}{2}+\sqrt{2}
$$

we show that any closed interval $[A ; B]$ of length $(1+\sqrt{5}) / 2+\sqrt{2}$ contains both conjugates of an algebraic integer of degree two. Without loss of generality, we assume that $A \in(-1 ; 0]$, since the intervals $[A ; B]$ and $[A+z ; B+z]$ with $z$ an integer either both contain or both do not contain any set of conjugates of an algebraic integer. There are three possibilities: $A \in(-1 ;(1-\sqrt{5}) / 2], A \in((1-\sqrt{5}) / 2 ; 1-\sqrt{2}]$ and $A \in(1-\sqrt{2} ; 0]$. In the first case we have

$$
B>\frac{1+\sqrt{5}}{2}+\sqrt{2}-1=\frac{\sqrt{5}-1}{2}+\sqrt{2}
$$

and $[A ; B]$ contains both roots of $x^{2}-x-1$.

In the second case,

$$
B>\frac{1+\sqrt{5}}{2}+\sqrt{2}+\frac{1-\sqrt{5}}{2}=1+\sqrt{2}
$$

and $[A ; B]$ contains both roots of $x^{2}-2 x-1$.

Finally, in the third case,

$$
B>\frac{1+\sqrt{5}}{2}+\sqrt{2}+1-\sqrt{2}=\frac{3+\sqrt{5}}{2}
$$

and $[A ; B]$ contains both roots of $x^{2}-3 x+1$. Theorem 1 is proved.

We now show how to compute $I(d)$ for "small" $d$. As we already noticed, there is no loss of generality to assume that the left endpoints of the intervals lie in $(-1 ; 0]$. So the right endpoinds are bounded above, say, by 5 . However the interval $(-1 ; 5)$ contains only a finite number of sets of conjugates of algebraic integers of degree $d$. Suppose there are $M$ such sets. Clearly, $M \geq 1$, since $[0 ; 4]$ contains such a set. Let also $\beta_{1}, \ldots, \beta_{M}$ be the smallest conjugates in these sets and let $\gamma_{1}, \ldots, \gamma_{M}$ be the largest ones. Since for $d \geq 2$ we have $I(d)>3$, the intersection of the intervals $\left[\beta_{i} ; \gamma_{i}\right]$ and $\left[\beta_{j} ; \gamma_{j}\right]$ is nonempty. So

$$
I(d)=\max \left\{\gamma_{j}-\beta_{i}\right\},
$$

where the maximum is taken over all $i, j, 1 \leq i, j \leq M$, such that for each $s$, $1 \leq s \leq M$, either $\beta_{s}<\beta_{i}$ or $\gamma_{s}>\gamma_{j}$. If $d$ is small, then $M$ is not very large. Having all $M$ polynomials of degree $d$ with all roots in $(-1 ; 5)$ one can apply formula (6) in order to find $I(d)$ explicitly. From (6) it is also clear that we can replace the word "closed" by "half-closed" (interval) in the definition of $I(d)$. 
This research was partially supported by a grant from Lithuanian Foundation of Studies and Science.

\section{References}

[Du] A. Dubickas, On the maximal conjugate of a totally real algebraic integer, Lithuanian Math. J. 37 (1997), 13-19.

[En] V. Ennola, Conjugate algebraic integers in an interval, Proc. Amer. Math. Soc. 53 (1975), 259-261.

[Kr] L. Kronecker, Zwei Sätze über Gleichungen mit ganzzahligen Coefficienten, J. Reine Angew. Math. 53 (1857), 173-175.

[La] M. Langevin, Solution des problèmes de Favard, Ann. Inst. Fourier (Grenoble) 38 (1988), no. 2, 1-10.

[Le] D. H. Lehmer, Factorization of certain cyclotomic functions, Ann. of Math. 34 (1933), 461-479.

[Lo] R. Louboutin, Sur la mesure de Mahler d'un nombre algébrique, C. R. Acad. Sci. Paris 296 (1983), 707-708.

[Ro] R. M. Robinson, Intervals containing infinitely many sets of conjugate algebraic integers, in: Studies in Mathematical Analysis and Related Topics, Stanford Univ. Press, 1962, 305-315.

[SZ] A. Schinzel and H. Zassenhaus, A refinement of two theorems of Kronecker, Michigan Math. J. 12 (1965), 81-85.

[Sc] I. Schur, Über die Verteilung der Wurzeln bei gewissen algebraischen Gleichungen mit ganzzahligen Koeffizienten, Math. Z. 1 (1918), 377-402.

[Za] T. Zaïmi, Minoration du diamètre d'un entier algébrique totalement réel, C. R. Acad. Sci. Paris 319 (1994), 417-419.

Department of Mathematics and Informatics

Vilnius University

Naugarduko 24

2600 Vilnius, Lithuania

E-mail: arturas.dubickas@maf.vu.lt

Received on 16.3.1999

and in revised form on 17.6.1999 